\title{
A NOTE ON SEMIGROUPS IN RINGS
}

\author{
STEVE LIGH
}

(Received 1 November, 1975; revised 20 December 1976)

Communicated by T. E. Hall

\section{Introduction}

Recently J. Cresp and R. P. Sullivan (1975) investigated those rings $R$ with the following properties:

(*) every multiplicative subsemigroup of $R$ is a subring.

$(* *)$ every multiplicative subsemigroup of $R$ containing 0 is a subring.

For rings with $(*)$ they obtained the following characterization.

Proposition 1. A ring $R$ has $(*)$ if and only if either $|R|=1$ or $|R|=2$ and $R^{2}=0$.

For rings with $(* *)$ they characterized all such rings with an identity by employing a result of Gluskin (1963).

Proposition 2. A ring $R$ containing an identity has $(* *)$ if and only if it is a finite field such that $|R-0|$ is a prime number.

The purpose of this note is to furnish a characterization of those rings with $(* *)$ without assuming an identity and the use of Gluskin's result. Also we will consider some generalizations.

\section{Subsemigroups of rings}

A subset $S$ of a ring $(R,+,$.$) will be called a subsemigroup of R$ if it is a subsemigroup of $(R,$.$) . As usual, for each x$ in $R,\langle x\rangle$ denotes the cyclic subsemigroup of $R$ generated by $x$. In this section we characterize completely those rings with property $(* *)$. Our theorem follows from a series of lemmas.

Lemma 1. Let $R$ be a ring with $(* *)$. If there is $e \neq 0$ in $R$ such that $e^{2}=e$, then $x+x=0$ for all $x$ in $R$.

Proof. Since $\{0, e\}$ is a subsemigroup, it follows that $e+e=0$. Hence 
$e x+e x=(e+e) x=0$ and $x e+x e=x(e+e)=0$ for all $x$ in $R$. Thus $\{0, e\} \cup\langle x+x\rangle$ is a subsemigroup of $R$, and since $R$ has $(* *)$, we see that $e+x+x$ equals $0, e$ or $(x+x)^{\prime}$ for some positive integer $j$. If $e+x+x=0$, then $x+x=e$ and $e x+e x=e^{2}=0$, a contradiction. If $e+x+x=(x+x)^{\prime}$, then $e=e(e+x+x)=e(x+x)^{j}=(e x+e x)(x+x)^{j-1}=0$, a contradiction. Thus the remaining case yields the fact the $e+x+x=e$ and $x+x=e+e=$ 0 .

Lemma 2. Let $R$ be a ring with $(* *)$. If there is $e^{2}=e \neq 0$ in $R$, then $e x=x e=x$ for all $x$ in $R$.

Proof. From Lemma $1, x+x=0$ for each $x$ in $R$. Suppose exe $+e x \neq 0$. Also exe $+e x \neq e$. For if not, exe $+e x=e$ implies that $0=e x e+e x e=e^{2}$. Thus $\{0, e\} \cup\langle e x e+e x\rangle$ is a subsemigroup and it follows that exe $+e x+e$ equals $0, e$ or $(e x e+e x)^{\prime}$ for some positive integer $j$. Now exe $+e x+e=0$ implies that $e x e+e x=e$ and $e x e+e x e=e^{2}=0$, a contradiction. Similarly, $e x e+e x+e=e$ implies that $e x e+e x=0$. Thus we must have that $e x e+e x+e=(e x e+e x)^{i}$. Hence $(e x e+e x+e) e=(e x e+e x)^{j-1}(e x e+e x e)$ $=0$. But this means that exe $+e x e+e=0$ and $e=0$, a contradiction. Hence $e x e+e x=0$ and $e x e=e x$. By a similar argument, exe $=x e$ and it follows that $e x=x e$ for each $x$ in $R$.

Next we wish to establish that $e x=x$ for each $x$ in $R$. Observe that $e x+x=e$ implies that $e x+e x=e^{2}=0$. Thus $e x+x \neq e$. Suppose $e x+x \neq 0$. Then $\{0, e\} \cup\langle e x+x\rangle$ is a subsemigroup. Again, $e x+x+e$ equals $0, e$ or $(e x+x)^{i}$. It can be checked as in the above argument that each of the three possibilities gives a contradiction. Thus we conclude that $e x+x=0$ and $e x=x$ for each $x$ in $R$.

LeMma 3. Let $R$ be a ring with $(* *)$. If there is an $x$ in $R$ such that for all positive integers $n, x^{n} \neq 0$, then there is an $e^{2}=e \neq 0$ in $R$.

Proof. Suppose $x^{n} \neq 0$ for each positive integer $n$. Then $x+x=0$. For if not, since $\{0\} \cup\langle x\rangle$ is a subsemigroup, $-x=x^{i}$ for some positive integer $j>1$. Hence $x^{2}=(-x)(-x)=x^{21}$. Thus there is an integer $k$ such that $\left(x^{k}\right)^{2}=x^{k}$. Thus $x^{k}=e=e^{2}$. But by Lemma $1, x+x=0$, a contradiction.

From above it follows that $x^{i}+x^{i}=0$ for each positive integer $i$. Again from the subsemigroup $\{0\} \cup\langle x\rangle$, we have that $x+x^{2}=x^{j}, j \geqq 3$ and observe that $\left(x+x^{j-1}\right) x=x=x\left(x+x^{j-1}\right)$, or $x+x^{2}=0$ (in which case we let $e=x$ ). Now we wish to show that $\left(x+x^{j-1}\right)$ is an idempotent: it follow from the calculation below. 


$$
\begin{aligned}
\left(x+x^{j-1}\right)\left(x+x^{j-1}\right) & =x^{2}+x^{j}+x^{j}+x^{j-1} x^{j-1} \\
& =x+x^{j-1}\left(x+x^{j-1}\right) \\
& =x+x^{j-1} .
\end{aligned}
$$

Now we are ready to state and prove our main result.

THEOREM 1. A ring $R$ has property $(* *)$ if and only if either $|R|=1$ or $R \mid=2$ and $R^{2}=0$ or $R$ is a finite field and $|R-0|$ is a prime number.

Proof. Suppose $R$ has $(* *)$ and there is an element $x \neq 0$ in $R$ such that $x^{n} \neq 0$ for each positive integer $n$. By Lemma 3 , there is an $e=e^{2} \neq 0$ in $R$ and by Lemma $2, e$ is the identity of $R$. By Proposition $2, R$ is a finite field and $|R-0|$ is a prime number. Now suppose $|R|>1$ and every $x \neq 0$ in $R$ is nilpotent. By following the proof of Theorem 1 by Cresp and Sullivan (1975) we see that $|R|=2$ and $R^{2}=0$.

The converse is immediate and thus the proof of the theorem is complete.

\section{Generalizations}

In this section we extend Propositions 1 and 2 to the class of near-rings and Gluskin's result (1963) will not be needed in one of the proofs (Theorem 3). For definitions and basic facts about near-rings, see Ligh (1969). Furthermore, replace "subring" by "sub-near-ring" in the definition of property (*) and $(* *)$.

THEOREM 2. A near-ring $R$ has property (*) if and only if either $|R|=1$ or $|R|=2$ and $R^{2}=0$.

Proof. Using a similar argument to the first part of the proof of Theorem 1 by Cresp and Sullivan (1975), we have that $x^{2}=0$ for each $x$ in $R$. Thus $0 x=(0 x)(0 x)=0$ and $\{0, x\}$ is a subsemigroup. It follows that $x+x=0$ for each $x$ in $R$ and $(R,+)$ is commutative.

Now suppose $x \neq 0$ and $y \neq 0$ are in $R$. Then $x(x+y)(x+y)=0$ implies $\left(x^{2}+x y\right)(x+y)=0$ and hence $x y x=0$. Thus $\{0, x, x y\}$ is a subsemigroup and by $(*), x+x y=0, x$ or $x y$. A quick calculation shows that $x y=0$. Similarly $y x=0$.

Now consider the subsemigroup $\{0, x, y\}$. It follows that $x+y=0$ and $x=y$. Hence $|R|=2$ and $R^{2}=0$.

THEOREM 3. Let $R$ be a near-ring with identity 1. If $R$ has property $(* *)$, then $R$ is a near-field. Furthermore, if $R$ is finite, then $R$ is a field such that $|R-0|$ is a prime number. 
Proof. Since $\{0,1\}$ is a subsemigroup, $1+1=0$ and hence $(R,+)$ is abelian. Suppose $x \neq 0,1$ is in $R$. Then $\{0,1\} \cup\langle x+1\rangle$ is a subsemigroup of $R$, and by $(* *), x=1+(1+x)=(1+x)^{j}$ for some $j \geqq 2$. On the other hand, $\{0,1\} \cup\langle x\rangle$ is also a subsemigroup, thus $(1+x)=x^{s}$ for some $s \geqq 2$. Hence there is a positive integer $n \geqq 2$ such that $x^{n}=x$ and suppose $n$ is the smallest. Again from the subsemigroup $\{0,1\} \cup\langle x\rangle$, we have that $\left(1+x^{n-1}\right)$ equals 0,1 or $x^{\prime}$. Since the second and third possibilities give a contradiction, it follows that $x^{n-1}=1$ and hence each $x \neq 0$ in $R$ has a multiplicative inverse and $R$ is therefore a near-field.

Suppose the near-field $R$ is finite. Since $1+1=0$ in $R, R$ has characteristic 2 and by Corollary 2 in Ligh, McQuarrie and Slotterbeck (1972), the order of $R$ is $2^{n}$ for some positive integer $n$. Let $2^{n}-1=p_{1}^{n_{i}} \cdots p_{j}^{n_{j}}$ where each $p_{i}$ is an odd prime. Since $(R-0,$.$) is a group, for each p_{i}^{n_{i}}$, there is a subgroup $S_{i}$ of order $p_{i}^{n_{i}}$. Thus the semigroup $0 \cup S_{i}$ has order $p_{i}^{n_{i}}+1=2^{m_{i}}$. By Theorem 1 in Ligh and Neal (1974), $n_{i}=1$ for each $i$. Hence

$$
2^{n}-1=\left(2^{m_{1}}-1\right)\left(2^{m_{2}}-1\right) \cdots\left(2^{m_{i}}-1\right) .
$$

By expanding the right-hand side of the above equation, one gets a contradiction if $j \geqq 2$. Thus $2^{n}-1=p$ and $(R-0,$.$) is a commutative group. It follows$ that $R$ is a field.

\section{References}

J. Cresp and R. P. Sullivan (1975), 'Semigroups in rings', J. Austral. Math. Soc. 20, $172-177$.

L. M. Gluskin (1963), 'Ideals in rings and their multiplicative semigroups', Uspedni Mat. Nauk. (N. S.) 15, 4 (94), 141-148; translated in Amer. Math. Soc. Translations, 27 (2) 297-304.

Steve Ligh (1969). "On boolean near-rings", Bull. Austral. Math. Soc. 1, 375-379.

Steve Ligh, Bruce McOuarrie and Oberta Slotterbeck (1972), 'On near-fields', J. London Math. Soc. (2) 5, 87-90.

Steve Ligh and Larry Neal (1974), 'A note on Mersenne numbers', Math. Magazine 47, 231-233.

Department of Mathematics,

University of Southwestern Louisiana,

Lafayette, Louisiana, USA. 